\title{
FANO VARIETIES WITH SMALL NON-KLT LOCUS
}

\author{
MAURO C. BELTRAMETTI, ANDREAS HÖRING, AND CARLA NOVELLI
}

\begin{abstract}
Let $X$ be a Fano variety of index $k$. Suppose that the non-klt locus $\operatorname{Nklt}(X)$ is not empty. We prove that $\operatorname{dim} \operatorname{Nklt}(X) \geq k-1$ and equality holds if and only if $\operatorname{Nklt}(X)$ is a linear $\mathbb{P}^{k-1}$. In this case $X$ has lc singularities and is a generalised cone with $\operatorname{Nklt}(X)$ as vertex.

If $X$ has lc singularities and $\operatorname{dim} \operatorname{Nklt}(X)=k$ we describe the non-klt locus $\operatorname{Nklt}(X)$ and the global geometry of $X$. Moreover, we construct examples to show that all the classification results are effective.
\end{abstract}

\section{INTRODUCTION}

Let $X$ be a Fano variety, i.e. $X$ is a normal projective complex variety such that the anticanonical divisor $-K_{X}$ is Cartier and ample. While Fano varieties with mild singularities (terminal or canonical) have been studied by many authors, the goal of this paper is to study Fano varieties whose non-klt locus $\operatorname{Nklt}(X)$ is not empty. A cone over a smooth variety $Y$ with trivial anticanonical divisor provides a simple example of such a variety. Using methods from the minimal model program, Ishii Ish91, Ish94 characterised cones as the only Fano varieties having a finite non-klt locus:

1.1. Theorem. Ish91 Let $X$ be a Fano variety of dimension $n$ such that $\operatorname{Nklt}(X)$ is not empty and finite. Then $X$ is a cone over a variety $Y$ of dimension $n-1$ such that $Y$ has canonical singularities and $K_{Y}$ is trivial.

Recall now that the index of a Fano variety $X$ is defined as

$$
\sup \left\{m \in \mathbb{N} \mid \exists H \in \operatorname{Pic}(X) \text { s.t. }-K_{X} \simeq m H\right\} .
$$

Making stronger assumptions on the singularities of $X$, the first-named author and Sommese established a basic relation between the index and the dimension of the non-klt locus:

1.2. Theorem. BS87 Let $X$ be a Fano variety of index $k$ that is Cohen-Macaulay and such that $-K_{X} \simeq k H$, with $H$ a very ample Cartier divisor on $X$. Suppose that the non-klt locus $\operatorname{Nklt}(X)$ is not empty. Then we have

$$
\operatorname{dim} \operatorname{Nklt}(X) \geq k-1,
$$

and equality holds if and only if $\operatorname{Nklt}(X)$ is a linear $\mathbb{P}^{k-1}$ and $X$ is a generalised cone with $\operatorname{Nklt}(X)$ as vertex.

Date: September 20, 2013.

2000 Mathematics Subject Classification. 14J45, 14E30, 14D06, 14J40, 14M22.

Key words and phrases. Fano variety, non-klt locus. 
Unfortunately the Cohen-Macaulay condition excludes an important number of examples: cones over Calabi-Yau manifolds are Cohen-Macaulay, but a cone over an abelian variety is not. Our first result generalises Theorem 1.1 and Theorem 1.2

1.3. Theorem. Let $X$ be a Fano variety of index $k$ and such that $-K_{X} \simeq k H$, with $H$ a Cartier divisor on $X$. Suppose that the non-klt locus $\operatorname{Nklt}(X)$ is not empty. Then we have

$$
\operatorname{dim} \operatorname{Nklt}(X) \geq k-1,
$$

and equality holds if and only if $\left(\operatorname{Nklt}(X), \mathcal{O}_{\text {Nklt }(X)}(H)\right) \cong\left(\mathbb{P}^{k-1}, \mathcal{O}_{\mathbb{P}^{k-1}}(1)\right)$. In this case $X$ has lc singularities and is a generalised cone with $\operatorname{Nklt}(X)$ as vertex.

A remarkable feature that is common to the Theorems 1.1, 1.2 and 1.3 is that the property $\operatorname{dim} \operatorname{Nklt}(X)=k-1$ implies that the singularities of $X$ are log-canonical. If $\operatorname{dim} \operatorname{Nklt}(X)=k$ this is no longer the case (cf. Example 2.2), so for the rest of the paper we will make the additional assumption that $X$ is a Fano variety with lc singularities. Since we assume $K_{X}$ to be Cartier the non-klt locus coincides with the locus of irrational singularities [KM98, Cor.5.24], so we can study $\operatorname{Nklt}(X)$ both in terms of discrepancies and using cohomological methods. Note also that Theorem 1.3 naturally separates into a local part, i.e. the description of the non-klt locus $\operatorname{Nklt}(X)$, and a global part, i.e. the description of the geometry of $X$. For the local part we use a subadjunction argument to describe the low-dimensional lc centres:

1.4. Theorem. Let $X$ be a Fano variety of index $k$ with lc singularities and such that $-K_{X} \simeq k H$, with $H$ a Cartier divisor on $X$. If $\operatorname{dim} \operatorname{Nklt}(X)=k$ we have

$$
\left(\operatorname{Nklt}(X), \mathcal{O}_{\operatorname{Nklt}(X)}(H)\right) \cong\left(\mathbb{P}^{k}, \mathcal{O}_{\mathbb{P}^{k}}(1)\right)
$$

or

$$
\left(\operatorname{Nklt}(X), \mathcal{O}_{\text {Nklt }(X)}(H)\right) \cong\left(Q^{k}, \mathcal{O}_{Q^{k}}(1)\right),
$$

where $Q^{k} \subset \mathbb{P}^{k+1}$ is a (possibly reducible) hyperquadric.

This local result gives some interesting global information:

1.5. Corollary. Let $X$ be a Fano variety of index $k$ with lc singularities and such that $\operatorname{dim} \operatorname{Nklt}(X)=k$. Then $X$ is rationally chain-connected.

Indeed by a result of Broustet and Pacienza BP11, Thm.1.2] the variety $X$ is rationally chain-connected modulo the non-klt locus. Since Nklt $(X)$ is rationally chain-connected the statement follows.

We continue the study of the case $\operatorname{dim} \operatorname{Nklt}(X)=k$ by considering a terminal modification $X^{\prime} \rightarrow X$ (cf. Definition 1.10). If $\operatorname{Nklt}(X)$ is a linear $\mathbb{P}^{1}$ the variety $X^{\prime}$ can be rationally connected and have a very rich birational geometry, cf. Ish91, Ish94. If $\operatorname{Nklt}(X)$ is a conic the variety $X^{\prime}$ is never rationally connected and we obtain a precise classification:

1.6. Theorem. Let $X$ be a Fano variety (of index 1 ) and dimension $n \geq 3$ with lc singularities. Suppose that $\operatorname{Nklt}(X)$ is a curve and $-K_{X} \cdot \operatorname{Nklt}(X)=2$. Let $\mu: X^{\prime} \rightarrow X$ be a terminal modification. Then the base of the MRC-fibration $X^{\prime} \rightarrow Z$ has dimension $n-2$ and the general fibre $F$ polarised by $\mathcal{O}_{F}\left(-\mu^{*} K_{X}\right)$ is a linear $\mathbb{P}^{2}$, a quadric, a Veronese surface or a ruled surface. 
In fact we know much more. The base $Z$ has an effective canonical divisor, moreover we can construct birational models of $X$ which have a very simple fibre space structure:

1.7. Proposition. Let $X$ be a Fano variety of dimension $n$ and index $k$ with $l c$ singularities such that $\operatorname{dim} \operatorname{Nklt}(X)=k$. Suppose that the base of the $M R C$-fibration $X^{\prime} \rightarrow Z$ has dimension $n-k-1$. Then there exists a normal projective variety $\Gamma$ admitting a birational morphism $p: \Gamma \rightarrow X$ and an equidimensional fibration $q: \Gamma \rightarrow \mathcal{H}$ onto a projective manifold $\mathcal{H}$ such that one of the following holds:

a) the fibration $q$ is a projective bundle;

b) the fibration $q$ is a quadric fibration;

c) the general $q$-fibre is a Veronese surface.

In Section 6 we show that all the classification results in this paper are effective, i.e. there exist examples realising all the cases in Theorem 1.6 and Proposition 1.7. The condition $\operatorname{dim} Z=n-k-1$ may seem rather ad-hoc, but we prove that it is satisfied if $\operatorname{Nklt}(X)$ is a quadric and $k=1$ or $k \geq n-3$ (cf. Proposition 5.4). Actually we prove that if $X$ admits a ladder (in the sense of Fujita, cf. Definition 5.21), then $H^{n-k-1}\left(X^{\prime}, \mathcal{O}_{X^{\prime}}\right) \neq 0$. We expect that any Fano variety with lc singularities admits a ladder, but this depends on the difficult non-vanishing conjecture Kaw00. Conj.2.1].

Acknowledgements. The first and the third authors thank the Research Network Program "GDRE-GRIFGA" for partial support. The second-named author was partially supported by the A.N.R. project "CLASS".

\section{Notation}

We work over the complex numbers, topological notions always refer to the Zariski topology. For general definitions we refer to Har77. The dimension of an algebraic variety is defined as the maximum of the dimension of its irreducible components.

On a normal variety we will denote by $\simeq$ the linear equivalence of Cartier divisors, while $\sim_{\mathbb{Q}}($ resp. $\equiv)$ will be used for the $\mathbb{Q}$-linear (resp. numerical) equivalence of $\mathbb{Q}$-Cartier $\mathbb{Q}$-divisors.

We will frequently use the terminology and results of the minimal model program (MMP) as explained in KM98, Deb01, Kol13. In particular klt stands for "Kawamata log terminal", dlt for "divisorial log terminal", plt for "purely log terminal", and lc for "log canonical" singularities.

1.8. Definition. Let $X$ be a normal variety. The canonical modification of $X$ is the unique projective birational morphism $\mu: X^{\prime} \rightarrow X$ from a normal variety $X^{\prime}$ with canonical singularities such that $K_{X^{\prime}}$ is $\mu$-ample.

1.9. Remarks. The existence of the canonical modification is a consequence of BCHM10, cf. the forthcoming book [Kol13]. If $K_{X}$ is $\mathbb{Q}$-Cartier we have

$$
K_{X^{\prime}} \sim_{\mathbb{Q}} \mu^{*} K_{X}-E
$$

where $E$ is an effective $\mathbb{Q}$-divisor whose support is the exceptional locus of $\mu$.

Let us recall that a normal variety is Gorenstein if it is Cohen-Macaulay and the canonical divisor is Cartier. Since canonical singularities are Cohen-Macaulay the 
first condition is empty for $X^{\prime}$, so the Gorenstein locus of $X^{\prime}$ is the open subset where $K_{X^{\prime}}$ is Cartier.

An important advantage of canonical models is their uniqueness, but their singularities can be rather complicated. One can improve the singularities by losing uniqueness:

1.10. Definition. Kol13 Let $X$ be a normal variety. A terminal modification of $X$ is a projective birational morphism $\mu: X^{\prime} \rightarrow X$ from a normal variety $X^{\prime}$ with terminal singularities such that $K_{X^{\prime}}$ is $\mu$-nef.

We will use the standard definitions and results of adjunction theory from Fuj90, BS95, except the following generalised version of the nefvalue of a Mori contraction:

1.11. Definition. Let $X$ be a normal quasi-projective variety with lc singularities, and let $H$ be a nef and big Cartier divisor on $X$. Let $\varphi: X \rightarrow Y$ be the contraction of a $K_{X}$-negative extremal ray $R$ such that $H \cdot R>0$. The nefvalue $r:=r(\varphi, H)$ is the positive number such that $\left(K_{X}+r H\right) \cdot R=0$.

Let us recall a well-known consequence of the cone theorem:

1.12. Lemma. Let $X$ be a normal projective variety with klt singularities such that

$$
-K_{X} \sim_{\mathbb{Q}} N+E
$$

where $N$ is a nef $\mathbb{Q}$-Cartier $\mathbb{Q}$-divisor and $E$ is a non-zero effective $\mathbb{Q}$-divisor. Suppose also that for every curve $C \subset X$ such that $N \cdot C=0$ we have $K_{X} \cdot C \geq 0$. Then there exists a $K_{X}$-negative extremal ray $R$ such that $E \cdot R>0$ and $N \cdot R>0$.

Proof. Arguing by contradiction we suppose that $-E \cdot R \geq 0$ for all $K_{X}$-negative extremal rays. If $C \subset X$ is a curve such that $K_{X} \cdot C \geq 0$, then

$$
-E \cdot C=K_{X} \cdot C+N \cdot C \geq 0 .
$$

By the cone theorem this implies that the antieffective divisor $-E$ is nef, a contradiction. The property $N \cdot R>0$ is trivial since $N$ is positive on all $K_{X}$-negative curves.

\section{Proof of Theorem 1.3}

Before we can prove Theorem 1.3 we need a technical lemma which replaces an inaccurate statement1 in And95, Thm.2.1(II,i)].

2.1. Lemma. Let $X$ be a normal quasi-projective variety with canonical singularities, and let $H$ be a nef and big Cartier divisor on $X$. Let $\varphi: X \rightarrow Y$ be a birational projective morphism with connected fibres onto a normal variety $Y$ such that $H$ is $\varphi$-ample and $K_{X}+r H$ is $\varphi$-numerically trivial for some $r>0$. Fix a point $y \in Y$ and suppose that all the irreducible components of $\varphi^{-1}(y)$ have dimension at most $\lfloor r\rfloor$. Suppose that there exists an irreducible component $F \subset \varphi^{-1}(y)$ that meets the Gorenstein locus of $X$. Then we have $\lfloor r\rfloor=r$.

\footnotetext{
${ }^{1}$ The statement is $\operatorname{dim} F \geq r$, but the proof only yields $\operatorname{dim} F \geq\lfloor r\rfloor$.
} 
Proof. The statement is local on the base, so we can assume that $Y$ is affine. In particular every $\varphi$-generated line bundle is globally generated.

By AW93, Thm.,p.740] we know that $H$ is $\varphi$-globally generated, moreover by And95, Thm.2.1(II,ii)] all the irreducible components of $\varphi^{-1}(y)$ are isomorphic to $\mathbb{P}^{\lfloor r\rfloor}$ and $\left.H\right|_{F}$ is a hyperplane divisor.

We will proceed by induction on $\lfloor r\rfloor$. For the case $\lfloor r\rfloor=1$ note that by Ish91, Lemma 1] we have $K_{X} \cdot F \geq-1$. Since $H \cdot F=1$ we obtain that $r \leq 1$. For the induction step choose $X^{\prime} \in|H|$ a general divisor, and consider the induced birational morphism $\left.\varphi\right|_{X^{\prime}}: X^{\prime} \rightarrow \varphi\left(X^{\prime}\right)$. Since $H$ is $\varphi$-globally generated the variety $X^{\prime}$ is normal with canonical singularities, moreover the fibre $\left.\varphi\right|_{X^{\prime}} ^{-1}(y)$ has pure dimension $\lfloor r\rfloor-1$ and $F \cap X^{\prime}$ meets the Gorenstein locus of $X^{\prime}$. By adjunction we know that $K_{X^{\prime}}+\left.(r-1) H\right|_{X^{\prime}}$ is $\left.\varphi\right|_{X^{\prime}}$-numerically trivial, so by the induction hypothesis we have $\lfloor r-1\rfloor=r-1$.

Proof of Theorem 1.3. Let $\mu: X^{\prime} \rightarrow X$ be the canonical modification of $X$ (cf. Definition 1.8). Then we have

$$
K_{X^{\prime}}=\mu^{*} K_{X}+\sum_{E_{i}} a_{\mu \text {-exc. }} a_{i} E_{i}
$$

and $a_{i} \leq-1$ for all $i$. Set $E:=-\sum_{E_{i} \mu \text {-exc. }} a_{i} E_{i}$, then $E$ is an effective divisor mapping onto $\operatorname{Nklt}(X)$. Since $K_{X}$ is Cartier, the non-Gorenstein locus of $X^{\prime}$ is contained in $E$.

By Lemma 1.12 there exists a $K_{X^{\prime}}$-negative extremal ray $R$ on $X^{\prime}$ such that $E \cdot R>0$ and $\mu^{*} H \cdot R>0$. Let $\varphi: X^{\prime} \rightarrow Y$ be the corresponding Mori contraction, and let $F$ be an irreducible component of a positive-dimensional $\varphi$-fibre. Since $E$ is $\varphi$-ample, the intersection $E \cap F$ is non-trivial. Since $E$ is $\mathbb{Q}$-Cartier we have

$$
\operatorname{dim}(F \cap E) \geq \operatorname{dim} F-1,
$$

and equality holds if and only if $F \not \subset E$. Since $K_{X}$ is $\mu$-ample and $\varphi$-antiample the morphism

$$
(F \cap E) \rightarrow \mu(F \cap E)
$$

is finite, thus we have

$$
\operatorname{dim} \operatorname{Nklt}(X)=\operatorname{dim} \mu(E) \geq \operatorname{dim} \mu(F \cap E)=\operatorname{dim}(F \cap E) \geq \operatorname{dim} F-1,
$$

and equality holds if and only if $\operatorname{dim} F=\operatorname{dim} \operatorname{Nklt}(X)+1$ and $F \not \subset E$.

Since $-K_{X^{\prime}} \sim_{\mathbb{Q}} k \mu^{*} H+E$ we see that the nefvalue $r:=r(\varphi, H)$ (cf. Definition 1.11) is strictly larger than $k$. By [And95, Thm.2.1(I,i)] this implies that $\operatorname{dim} F \geq$ $r-1>k-1$. By (11) we get $\operatorname{dim} \operatorname{Nklt}(X)>k-2$. Since $\operatorname{dim} \operatorname{Nklt}(X)$ is an integer the inequality in the statement follows.

Suppose now that we are in the boundary case $\operatorname{dim} \operatorname{Nklt}(X)=k-1$. Arguing by contradiction we suppose that the extremal contraction $\varphi$ is birational. By And95. Thm.2.1(II,i)] we then have $\operatorname{dim} F \geq\lfloor r\rfloor \geq k$. Thus equality holds in (1) and the variety $F$ is not contained in $E$. Therefore we satisfy the conditions of Lemma 2.1 and get $\lfloor r\rfloor=r$, so $r=k$ by (11). However by construction we have $r>k$, a contradiction.

Thus the contraction $\varphi$ is of fibre type; moreover all the irreducible components of each $\varphi$-fibre have dimension exactly $k$. By And95, Thm.2.1(I,ii)] this implies 
that $X^{\prime} \rightarrow Y$ is a $\mathbb{P}^{k}$-bundle. Let now $F \cong \mathbb{P}^{k}$ be a general $\varphi$-fibre. Then $F$ is contained in the smooth locus of $X^{\prime}$, in particular all the divisors $E_{i}$ are Cartier in a neighborhood of $F$. By adjunction we see that

$$
\mathcal{O}_{F}\left(\sum a_{i} E_{i}\right) \simeq \mathcal{O}_{\mathbb{P}^{k}}(-1)
$$

Since the left hand side is a sum of antieffective Cartier divisors, we see that (up to renumbering) we have $a_{1}=-1$ and $E_{1} \cap F$ is a hyperplane. Moreover we have $E_{i} \cap F=\emptyset$ for all $i \geq 2$. In particular for every $i \geq 2$ we have $E_{i} \simeq \varphi^{*} D_{i}$ with $D_{i}$ a Weil divisor on $Y$. However if we take $y \in D_{i}$ a point, then $\varphi^{-1}(y) \subset E_{i}$ and $-\left.K_{X^{\prime}}\right|_{F}$ is ample, so $F \rightarrow \mu(F) \subset \mu\left(E_{i}\right)$ is finite. Since we assumed that $\operatorname{dim} \operatorname{Nklt}(X)=\operatorname{dim} \mu(E)=k-1$ this yields $E_{i}=0$ for all $i \geq 2$. Thus we have

$$
K_{X^{\prime}}=\mu^{*} K_{X}-E_{1}
$$

One easily sees that the pair $\left(X^{\prime}, E_{1}\right)$ is lc, so $X$ has lc singularities. The generalised cone structure is given by the maps $\mu$ and $\varphi$.

2.2. Example. Let $Y \subset \mathbb{P}^{3}$ be a cone over a smooth quartic curve in $\mathbb{P}^{2}$, then $K_{Y}$ is trivial and the vertex is the unique irrational point on $Y$. Clearly $Y$ does not have lc singularities, so if $X$ is the cone over $Y$, then $X$ is a Fano variety of index one such that $\operatorname{Nklt}(X)$ has dimension one and $X$ is not lc.

\section{Description of the NON-KLT LOCUS}

Let $X$ be a normal quasi-projective variety, and let $\Delta$ be an effective boundary divisor on $X$ such that $K_{X}+\Delta$ is $\mathbb{Q}$-Cartier and the pair $(X, \Delta)$ is lc. Let $\operatorname{Nklt}(X, \Delta)$ be the non-klt locus. Recall that a subvariety $W \subset X$ is an lc centre of the pair $(X, \Delta)$ if there exists a birational morphism $\mu: X^{\prime} \rightarrow X$ and an effective divisor $E \subset X^{\prime}$ of discrepancy -1 such that $\mu(E)=W$. Recall also that the intersection $W_{1} \cap W_{2}$ of two lc centres $W_{i}$ is a union of lc centres [Kaw97, Prop.1.5]. In particular, given a point $x \in \operatorname{Nklt}(X, \Delta)$, there exists a unique lc centre $W$ passing through $x$ that is minimal with respect to the inclusion. By Kaw97, Thm.1.6] the lc centre $W$ is normal in the point $x$. If $W$ is minimal (in every point $x \in W$ ), the Kawamata subadjunction formula holds [Kaw98, FG12]: there exists an effective $\mathbb{Q}$-divisor $\Delta_{W}$ on $W$ such that the pair $\left(W, \Delta_{W}\right)$ is klt and

$$
K_{W}+\left.\Delta_{W} \sim_{\mathbb{Q}}\left(K_{X}+\Delta\right)\right|_{W} .
$$

The following weak form of the subadjunction formula for non-minimal lc centres is well-known to experts, we nevertheless include a proof for lack of reference:

3.1. Lemma. Let $(X, \Delta)$ be a projective lc pair, and let $W \subset X$ be an lc centre. Let $\nu: W^{n} \rightarrow W$ be the normalisation. Then there exists an effective divisor $\Delta_{W^{n}}$ on $W^{n}$ such that

$$
K_{W^{n}}+\left.\Delta_{W^{n}} \sim_{\mathbb{Q}} \nu^{*}\left(K_{X}+\Delta\right)\right|_{W} .
$$

Suppose that $Z \subset W$ is an lc centre such that $\operatorname{dim} Z=\operatorname{dim} W-1$. Then we have a set-theoretical inclusion

$$
\nu^{-1}(Z) \subset \Delta_{W^{n}}
$$


Proof. Let $\mu:\left(X^{m}, \Delta^{m}\right) \rightarrow(X, \Delta)$ be a dlt-model, i.e. $\mu$ is birational, the pair $\left(X^{m}, \Delta^{m}\right)$ is dlt and $K_{X^{m}}+\Delta^{m} \sim_{\mathbb{Q}} \mu^{*}\left(K_{X}+\Delta\right)$ [KK10, Thm.3.1]. Let $S \subset X^{m}$ be an lc centre of $\left(X^{m}, \Delta^{m}\right)$ that dominates $W$ and that is minimal with respect to the inclusion. By Kol11, Thm.1] there exists an effective divisor $\Delta_{S}$ such that $\left(S, \Delta_{S}\right)$ is dlt and $K_{S}+\left.\Delta_{S} \sim_{\mathbb{Q}} \mu_{S}^{*}\left(K_{X}+\Delta\right)\right|_{W}$, where $\mu_{S}: S \rightarrow W$ is the restriction of $\mu$ to $S$. Moreover $\left(S, \Delta_{S}\right)$ is klt on the generic fibre of $\mu_{S}$. The variety $S$ being normal, the morphism $\mu_{S}$ factors through the normalisation $\nu$, and we denote by $\mu_{S}^{n}: S \rightarrow W^{\prime}$ and $\tau: W^{\prime} \rightarrow W^{n}$ the Stein factorisation.

Moreover,

$$
K_{S}+\left.\Delta_{S} \sim_{\mathbb{Q}}\left(\mu_{S}^{n}\right)^{*} \circ \tau^{*} \circ \nu^{*}\left(K_{X}+\Delta\right)\right|_{W}
$$

implies that $\mu_{S}^{n}$ is an lc-trivial fibration in the sense of Amb04, Defn.2.1] and we denote by $\Delta_{W^{\prime}}$ the discriminant divisor. Up to replacing $\mu_{S}^{n}$ by a birationally equivalent fibration we know by inversion of adjunction [Amb04, Thm.3.1] that the pair $\left(W^{\prime}, \Delta_{W^{\prime}}\right)$ is lc.

Using the terminology of Amb05 (see in particular Definition 3.2 and Theorem 3.3) the moduli b-divisor of the lc-trivial fibration is $b$-nef and good, in particular it has non-negative Kodaira dimension. Thus there exists an effective divisor $E$ such that

$$
K_{S}+\Delta_{S} \sim_{\mathbb{Q}}\left(\mu_{S}^{n}\right)^{*}\left(K_{W^{\prime}}+\Delta_{W^{\prime}}+E\right) .
$$

By the proof of [FG12, Lemma 1.1] there exists an effective divisor $\Delta_{W^{n}}$ such that

$$
K_{W^{\prime}}+\Delta_{W^{\prime}}+E \sim_{\mathbb{Q}} \tau^{*}\left(K_{W^{n}}+\Delta_{W^{n}}\right) .
$$

Recalling (3) we derive

$$
K_{W^{n}}+\left.\Delta_{W^{n}} \sim_{\mathbb{Q}} \nu^{*}\left(K_{X}+\Delta\right)\right|_{W} .
$$

Suppose now that $Z \subset W$ is an lc centre such that $\operatorname{dim} Z=\operatorname{dim} W-1$. Then we know by [Kol11, Cor.11] that every irreducible component of $(\nu \circ \tau)^{-1}(Z)$ is an lc centre of the pair $\left(W^{\prime}, \Delta_{W^{\prime}}\right)$. Since $(\nu \circ \tau)^{-1}(Z)$ is a divisor in $W^{\prime}$ we have a set-theoretical inclusion

$$
(\nu \circ \tau)^{-1}(Z) \subset \Delta_{W^{\prime}} .
$$

Since the pair $\left(W^{n}, \Delta_{W^{n}}\right)$ is not klt in the points where the pair $\left(W^{\prime}, \Delta_{W^{\prime}}+E\right)$ is not klt (cf. Kwc92, Prop.20.3]), the inclusion (4) implies a set-theoretical inclusion $\nu^{-1}(Z) \subset \Delta_{W^{n}}$.

For the description of the non-klt locus we start with a refinement of the local part of Theorem 1.3 in the log-canonical case:

3.2. Lemma. Let $(X, \Delta)$ be a projective lc pair such that $-\left(K_{X}+\Delta\right) \sim_{\mathbb{Q}} k H$, with $H$ an ample Cartier divisor and $k \geq 1$. Let $W \subset X$ be an lc centre. Then we have

$$
\operatorname{dim} W \geq k-1 \text {, }
$$

and equality holds if and only if $\lfloor k\rfloor=k$ and $\left(W,\left.H\right|_{W}\right) \cong\left(\mathbb{P}^{k-1}, \mathcal{O}_{\mathbb{P}^{k-1}}(1)\right)$.

Proof. The statement is trivial if $k=1$, so suppose $k>1$. It is sufficient to prove the statement for $W \subset X$ a minimal centre. By the subadjunction formula (2) there exists a divisor $\Delta_{W}$ on $W$ such that $\left(W, \Delta_{W}\right)$ is klt and

$$
K_{W}+\left.\Delta_{W} \sim_{\mathbb{Q}}\left(K_{X}+\Delta\right)\right|_{W} \sim_{\mathbb{Q}}-\left.k H\right|_{W} .
$$


If $\operatorname{dim} W>0$ we can apply [AD12, Thm.2.5] to see that the $\log$ Fano variety $\left(W, \Delta_{W}\right)$ has dimension at least $k-1$ and equality holds if and only if $\left(W,\left.H\right|_{W}\right) \cong$ $\left(\mathbb{P}^{k-1}, \mathcal{O}_{\mathbb{P}^{k-1}}(1)\right)$. Thus we are left to exclude the possibility that $\operatorname{dim} W=0$ : since $-H-\left(K_{X}+\Delta\right) \sim_{\mathbb{Q}}(k-1) H$ is ample, the restriction map

$$
H^{0}\left(X, \mathcal{O}_{X}(-H)\right) \rightarrow H^{0}\left(W, \mathcal{O}_{W}(-H)\right)
$$

is surjective by [Fuj11, Thm.2.2]. If $W$ is a point, the space $H^{0}\left(W, \mathcal{O}_{W}(-H)\right)$ is not zero, which is impossible since the antiample divisor $-H$ has no global sections on $X$.

The following proposition is the key step in the proof of Theorem 1.4

3.3. Proposition. Let $(X, \Delta)$ be a projective lc pair of dimension $n \geq 3$ such that $-\left(K_{X}+\Delta\right) \sim_{\mathbb{Q}} k H$, with $H$ an ample Cartier divisor and $k \in \mathbb{N}$. Let $W$ be an $l c$ centre of $(X, \Delta)$ of dimension $k$.

If $W$ is minimal, then $\left(W,\left.H\right|_{W}\right) \cong\left(\mathbb{P}^{k}, \mathcal{O}_{\mathbb{P}^{k}}(1)\right)$ or $\left(W,\left.H\right|_{W}\right) \cong\left(Q^{k}, \mathcal{O}_{Q}(1)\right)$, with $Q^{k} \subset \mathbb{P}^{k+1}$ an integral quadric.

If $W$ is not minimal, then $\left(W,\left.H\right|_{W}\right) \cong\left(\mathbb{P}^{k}, \mathcal{O}_{\mathbb{P}^{k}}(1)\right)$ and $W$ contains exactly one lc centre $Z \subsetneq W$.

Proof. If $W$ is minimal we know by the subadjunction formula (2) that there exists an effective divisor $\Delta_{W}$ on $W$ such that $\left(W, \Delta_{W}\right)$ is $\log$ Fano of index $k$ and dimension $k$. The statement then follows from [AD12, Thm.2.5].

Suppose now that $W$ is not minimal. Then $W$ contains another lc centre $Z \subsetneq W$ and by Lemma 3.2 we know that $Z$ has dimension $k-1$. Denote by $\nu: W^{n} \rightarrow W$ the normalisation. By Lemma 3.1 there exists an effective divisor $\Delta_{W^{n}}$ such that

$$
K_{W^{n}}+\left.\Delta_{W^{n}} \sim_{\mathbb{Q}} \nu^{*}\left(K_{X}+\Delta\right)\right|_{W} \sim_{\mathbb{Q}}-\left.k \nu^{*} H\right|_{W} .
$$

Thus the pair $\left(W^{n}, \Delta_{W^{n}}\right)$ is log Fano of dimension $k$ and index $k$, moreover by the last part of Lemma 3.1 we have a set-theoretical inclusion

$$
\nu^{-1}(Z) \subset \Delta_{W^{n}} .
$$

In particular $\Delta_{W^{n}}$ is not empty, so by [AD12, Thm.2.5] we obtain $W^{n} \cong \mathbb{P}^{k}$ and $\mathcal{O}_{W^{n}}\left(\nu^{*} H\right) \simeq \mathcal{O}_{\mathbb{P}^{k}}(1)$. By (5) this implies $\mathcal{O}_{W^{n}}\left(\Delta_{W^{n}}\right) \simeq \mathcal{O}_{\mathbb{P}^{k}}(1)$, so we see that $\nu^{-1}(Z)=\Delta_{W^{n}}$ and $\nu^{-1}(Z)$ is a hyperplane. Note that this already implies that $Z$ is unique.

Therefore it remains to prove that $W$ is normal. Note first that $W$ is minimal, hence normal, in the complement of $Z$. Thus it is sufficient to prove that $W$ is normal in every point $x \in Z$. By Lemma 3.2 we have $Z \cong \mathbb{P}^{k-1}$, so we get a finite map

$$
\left.\nu\right|_{\nu^{-1}(Z)}: \nu^{-1}(Z) \cong \mathbb{P}^{k-1} \rightarrow Z \cong \mathbb{P}^{k-1} .
$$

Since $\mathcal{O}_{\nu^{-1}(Z)}\left(\nu^{*} H\right) \simeq \mathcal{O}_{\mathbb{P}^{k-1}}(1)$ this map has degree one, so it is an isomorphism. This proves that the normalisation $\nu$ is an injection on points. By a result of Ambro Amb11, Thm.1.1] we know that $W$ is semi-normal, so $\nu$ is an isomorphism.

Proof of Theorem 1.4. By Nadel's vanishing theorem (see e.g. Fuj11, Thm.3.2]) we have $H^{1}\left(X, \mathcal{I}_{\mathrm{Nklt}(X)}\right)=0$, so the map

$$
H^{0}\left(X, \mathcal{O}_{X}\right) \rightarrow H_{8}^{0}\left(\operatorname{Nklt}(X), \mathcal{O}_{\text {Nklt }(X)}\right)
$$


is surjective. In particular the non-klt $\operatorname{locus} \operatorname{Nklt}(X)$ is connected. If $\operatorname{Nklt}(X)$ is irreducible we conclude by Proposition 3.3

Suppose from now on that $\operatorname{Nklt}(X)$ is reducible. If $W_{1}$ (resp. $W_{2}$ ) is an irreducible component of $\operatorname{Nklt}(X)$, hence an lc centre, of dimension $r_{1}$ (resp. $r_{2}$ ), the intersection $W_{1} \cap W_{2}$ is either empty or a union of lc centres of dimension at most $\min \left(r_{1}, r_{2}\right)-1$. By the connectedness of $\operatorname{Nklt}(X)$ we can reduce to consider the second case. Then Lemma 3.2 implies that every irreducible component of $\operatorname{Nklt}(X)$ has dimension $k$; moreover two components meet along a set of dimension $k-1$. By Lemma 3.3 every irreducible component $W_{i}$ is isomorphic to $\mathbb{P}^{k}$ and contains exactly one lc centre, so we see that $\operatorname{Nklt}(X)$ has exactly two irreducible components. These two irreducible components meet along an lc centre of dimension $k-1$, so by Lemma 3.2 the intersection $W_{1} \cap W_{2}$ is a linear $\mathbb{P}^{k-1}$. Thus $\operatorname{Nklt}(X)=W_{1} \cup W_{2}$ is a reducible quadric of dimension $k$.

\section{Description of the Geometry of the FAno VARIETy}

We start by classifying certain weak log Fano varieties that will appear as the fibres of the MRC-fibration:

4.1. Proposition. Let $X$ be a normal projective variety of dimension $k+1 \geq 2$ with terminal singularities, and let $\Delta$ be a non-zero effective Weil $\mathbb{Q}$-Cartier divisor on $X$ such that the pair $(X, \Delta)$ is $l c$, and

$$
-\left(K_{X}+\Delta\right) \sim_{\mathbb{Q}} k H
$$

with $H$ a nef and big Cartier divisor on $X$. Suppose also that for every curve $C \subset X$ such that $H \cdot C=0$ we have $K_{X} \cdot C \geq 0$. Then $\left(X, \mathcal{O}_{X}(H)\right)$ is one of the following quasi-polarised varieties:

a) $\left(\mathbb{P}^{k+1}, \mathcal{O}_{\mathbb{P}^{k+1}}(1)\right)$ and $\Delta$ is a quadric; or

b) $\left(Q^{k+1}, \mathcal{O}_{Q^{k+1}}(1)\right)$ and $\Delta$ is a quadric; or

c) a generalised cone of dimension $k+1$ over the Veronese surface $\left(\mathbb{P}^{2}, \mathcal{O}_{\mathbb{P}^{2}}(2)\right)$ and $\Delta$ is the generalised cone of dimension $k$ over $\left(\mathbb{P}^{1}, \mathcal{O}_{\mathbb{P}^{1}}(2)\right)$; or

d) a scroll $\mathbb{P}\left(\mathcal{O}_{\mathbb{P}^{1}}(a) \oplus \mathcal{O}_{\mathbb{P}^{1}}(1) \oplus \mathcal{O}_{\mathbb{P}^{1}}^{\oplus k-1}\right)$ where $a \in \mathbb{N}_{>0}$ and $\Delta$ is the union of $\mathbb{P}\left(\mathcal{O}_{\mathbb{P}^{1}}(1) \oplus \mathcal{O}_{\mathbb{P}^{1}}^{\oplus k-1}\right)$ and $a \mathbb{P}^{k} ;$ or

e) a scroll $\mathbb{P}\left(\mathcal{O}_{\mathbb{P}^{1}}(a) \oplus \mathcal{O}_{\mathbb{P}^{1}}(1)^{\oplus 2} \oplus \mathcal{O}_{\mathbb{P}^{1}}^{\oplus k-2}\right)$ where $a \in \mathbb{N}_{>0}$ and $\Delta=\mathbb{P}\left(\mathcal{O}_{\mathbb{P}^{1}}(1)^{\oplus 2} \oplus\right.$ $\left.\mathcal{O}_{\mathbb{P}^{1}}^{\oplus k-2}\right)$; or

f) a scroll $\mathbb{P}\left(\mathcal{O}_{\mathbb{P}^{1}}(a) \oplus \mathcal{O}_{\mathbb{P}^{1}}(2) \oplus \mathcal{O}_{\mathbb{P}^{1}}^{\oplus k-1}\right)$ where $a \in \mathbb{N}_{>0}$ and $\Delta=\mathbb{P}\left(\mathcal{O}_{\mathbb{P}^{1}}(2) \oplus\right.$ $\left.\mathcal{O}_{\mathbb{P 1}}^{\oplus k-1}\right)$; or

g) a scroll $\mathbb{P}(V)$ over an elliptic curve and $\Delta=\mathbb{P}(W)$ where $V \rightarrow W$ is a quotient bundle of rank $k$ such that $\operatorname{det} W \simeq \mathcal{O}_{W}$.

Proof. By Lemma 1.12 there exists a $K_{X}$-negative extremal ray $R$ such that $\Delta \cdot R>$ 0 and $H \cdot R>0$. Thus if $\varphi: X \rightarrow Y$ denotes the contraction of this extremal ray, the nefvalue $r:=r(\varphi, H)$ (cf. Definition 1.11) is strictly larger than $k$. Arguing by contradiction we suppose that the extremal contraction $\varphi$ is birational. Let $F$ be a non-trivial $\varphi$-fibre, then by [And95, Thm.2.1(II,i)] we have $\operatorname{dim} F \geq\lfloor r\rfloor \geq$ $k=\operatorname{dim} X-1$. Thus $\varphi$ contracts the divisor $F$ onto a point, in particular $F$ meets the Gorenstein locus of $X$. By Lemma 2.1 this implies that $r=\lfloor r\rfloor=k$, a 
contradiction. Thus $\varphi$ is of fibre type and we can apply [BS95, Sect.7.2, 7.3] to see that $X$ is isomorphic to one of the following varieties:

a) $\left(\mathbb{P}^{k+1}, \mathcal{O}_{\mathbb{P}^{k+1}}(1)\right)$; or

b) $\left(Q^{k+1}, \mathcal{O}_{Q^{k+1}}(1)\right)$; or

c) a generalised cone over $\left(\mathbb{P}^{2}, \mathcal{O}_{\mathbb{P}^{2}}(2)\right)$; or

d) a scroll over a curve $C$.

In the cases a)-c) we are obviously finished, so suppose that $X \cong \mathbb{P}(V)$ with $V$ a vector bundle of rank $k+1$. Note that $\Delta$ has exactly one irreducible component $\Delta_{1}$ that surjects onto $C$. Since $\Delta_{1} \rightarrow C$ is flat and every fibre is a hyperplane in $\mathbb{P}^{k}$, we see that $\Delta_{1} \cong \mathbb{P}(W)$ with $V \rightarrow W$ a quotient bundle of rank $k$. Set $\Delta^{\prime}:=\Delta-\Delta_{1}$. By the adjunction formula

$$
-\left(K_{\Delta_{1}}+\left(\Delta_{1} \cap \Delta^{\prime}\right)\right) \simeq-\left.k H\right|_{\Delta_{1}}
$$

and by the canonical bundle formula

$$
K_{\Delta_{1}}=\left.\varphi\right|_{\Delta_{1}} ^{*}\left(K_{C}+\operatorname{det} W\right)-\left.k H\right|_{\Delta_{1}}
$$

thus we obtain $-\left(\Delta_{1} \cap \Delta^{\prime}\right)=\left.\varphi\right|_{\Delta_{1}} ^{*}\left(K_{C}+\operatorname{det} W\right)$. Since $\operatorname{det} W$ is nef, this implies that $K_{C}$ is antinef. If $C$ is a rational curve we obtain the cases d)-f). If $C$ is an elliptic curve, the divisors $-\left(\Delta_{1} \cap \Delta^{\prime}\right)$ and $\operatorname{det} W$ must be trivial. Thus we obtain the case $g$ ).

4.2. Proposition. Let $X$ be a Fano variety of dimension $n$ and index $k$ with $l c$ singularities such that $-K_{X} \simeq k H$, with $H$ an ample Cartier divisor on $X$. Assume that $\operatorname{dim} \operatorname{Nklt}(X)=k$. Let $\mu: X^{\prime} \rightarrow X$ be a terminal modification of $X$, and write

$$
K_{X^{\prime}} \simeq \mu^{*} K_{X}-E,
$$

where $E$ is an effective, reduced, $\mu$-exceptional Weil divisor. Suppose that the base of the $M R C$-fibration $X^{\prime} \rightarrow Z$ has dimension $n-k-1$, and let $F$ be a general fibre. Then the log-pair $(F, F \cap E)$ quasi-polarised by the nef and big divisor $\left.\left(\mu^{*} H\right)\right|_{F}$ is isomorphic to one of the varieties a)-f) in Proposition 4.1. Moreover this statement is effective, i.e. there exist examples realising all these cases.

Proof. Since $X$ has lc singularities and $K_{X^{\prime}}+E \sim_{\mathbb{Q}} \mu^{*} K_{X}$, the pair $\left(X^{\prime}, E\right)$ is lc. By hypothesis $\operatorname{dim} Z=n-k-1$, so the general fibre $F$ is a $(k+1)$-dimensional variety with terminal singularities. Moreover the pair $\left(F,\left.E\right|_{F}\right)$ is lc and $\left.E\right|_{F} \neq 0$ since otherwise $X$ is not rationally connected modulo the non-klt locus, in contradiction to [BP11, Thm.1.2]. By adjunction we have $K_{F}+\left.E\right|_{F} \sim_{\mathbb{Q}}-\left.k\left(\mu^{*} H\right)\right|_{F}$, moreover any curve $C \subset F$ such that $\left.\left(\mu^{*} H\right)\right|_{F} \cdot C=0$ is $\mu$-exceptional, so $K_{F} \cdot C=K_{X^{\prime}} \cdot C \geq 0$. Thus Proposition 4.1 applies. Case g) is excluded since $F$ is rationally connected.

The statement is effective, the examples corresponding to the varieties a)-f) are: Examples 6.2 6.3, 6.4 6.5 and 6.6 (twice).

Remark. An analogous statement of Proposition 4.2 should hold if we replace a terminal modification by the canonical modification. However this would make it necessary to prove Proposition 4.1 and thus BS95, Sect.7.2, 7.3] for varieties with canonical singularities, a rather tedious exercise.

The following lemma is an analogue of classical descriptions of projective bundles as in BS95, Prop.3.2.1]: 
4.3. Lemma. Let $Z$ be a projective manifold, and let $X$ be a normal projective variety admitting an equidimensional fibration $\varphi: X \rightarrow Z$ of relative dimension $k$. Assume that there exists an ample Cartier divisor $H$ on $X$ such that the general polarised fibre $\left(F, \mathcal{O}_{F}(H)\right)$ is isomorphic to the quadric $\left(Q^{k}, \mathcal{O}_{Q^{k}}(1)\right)$. Then $X \rightarrow$ $Z$ is a quadric fibration, 2 , i.e. there exists a Cartier divisor $M$ on $Z$ such that $K_{X}+k H \sim_{\mathbb{Q}} \varphi^{*} M$

Proof. Let $C \subset Z$ be a complete intersection of $\operatorname{dim} Z-1$ general hyperplane sections. The preimage $X_{C}:=\varphi^{-1}(C)$ is a normal projective variety and the fibration $\varphi_{C}: X_{C} \rightarrow C$ satisfies the conditions of [Hör13, Lemma 2.6]. Thus we know that $X_{C}$ has canonical singularities, and there exists a Cartier divisor $M_{C}$ on $C$ such that

$$
K_{X_{C}}+\left.k H\right|_{X_{C}} \sim_{\mathbb{Q}} \varphi_{C}^{*} M_{C} .
$$

Using the canonical modification of $X$ we see that there exists a closed (maybe empty) subset $Z^{\prime} \subset Z$ of codimension at least two in $Z$ such that $X_{0}:=\varphi^{-1}\left(Z \backslash Z^{\prime}\right)$ has canonical singularities, and $\left.\left(K_{X}-k H\right)\right|_{X_{0}}$ is nef with respect to the equidimensional fibration $\varphi_{0}: X_{0} \rightarrow Z_{0}:=Z \backslash Z^{\prime}$. It follows from Zariski's lemma BHPVdV04, Lemma 8.2] that

$$
\left.\left(K_{X}+k H\right)\right|_{X_{0}} \sim_{\mathbb{Q}} \varphi_{0}^{*} M_{0}
$$

where $M_{0}$ is a Cartier divisor on $Z_{0}$. Since $Z$ is smooth the divisor $M_{0}$ extends to a Cartier divisor $M$ on $Z$. Since $X \backslash X_{0}$ has codimension at least two in $X$, the isomorphism above extends to $K_{X}+k H \sim_{\mathbb{Q}} \varphi^{*} M$.

Proof of Proposition 1.7. By hypothesis the base of the MRC-fibration $X^{\prime} \rightarrow Z$ has dimension $n-k-1$. Thus Proposition 4.2 applies, and the general fibre $F$ is given by the cases a)-f) of Proposition 4.1

If we are in case a), let $\mathcal{H}$ be a desingularisation of the unique component of the cycle space $\mathcal{C}(X)$ such that the general point parametrises $\mu(F)$, and let $q: \Gamma \rightarrow \mathcal{H}$ be the normalisation of the pull-back of the universal family. By construction the natural morphism $p: \Gamma \rightarrow X$ is birational and $p^{*} H$ is $q$-ample and its restriction to the general fibre is the hyperplane divisor. By [AD12, Prop.4.10], [HN13, Prop.3.5] the fibration $q$ is a projective bundle. If we are in the cases d)-f) the MRC-fibration factors generically through an almost holomorphic fibration $X^{\prime} \rightarrow W$ such that the general fibre is a linear $\mathbb{P}^{k-1}$, so we use the same argument to construct a $\mathbb{P}^{k-1}$-bundle $\Gamma \rightarrow \mathcal{H}$ that dominates $X$.

If we are in case b) let $\mathcal{H}$ be a desingularisation of the unique component of the cycle space $\mathcal{C}(X)$ such that the general point parametrises $\mu(F)$, and let $q: \Gamma \rightarrow \mathcal{H}$ be the normalisation of the pull-back of the universal family. Then $q$ is a quadric fibration by Lemma 4.3 .

If we are in case c) and $k=1$ the general fibre of the MRC-fibration is the Veronese surface. We repeat the construction above to obtain $\mathcal{H}$ and $\Gamma \rightarrow \mathcal{H}$. If $k \geq 2$ the $(k+1)$-dimensional cone over the Veronese surface is dominated by the projective bundle $\mathbb{P}\left(\mathcal{O}_{\mathbb{P}^{2}}(2) \oplus \mathcal{O}_{\mathbb{P}^{2}}^{\oplus k-1}\right)$. Thus we can repeat the construction of case a) to obtain a $\mathbb{P}^{k-1}$-bundle $\Gamma \rightarrow \mathcal{H}$ that dominates $X$.

\footnotetext{
${ }^{2}$ We use the definition of an adjunction theoretic quadric fibration [BS95. 3.3.1] which is a-priori weaker than supposing that all the fibres are quadrics.
} 


\section{ThE BASE OF THE MRC-FiBRATION}

Proposition 1.7 gives a rather precise description of the Fano variety $X$, but the condition on the MRC-fibration seems rather restrictive. In this section we will give strong evidence that this condition is always satisfied when $\operatorname{Nklt}(X)$ is a quadric, in particular we prove Theorem 1.6.

The following lemma generalises a part of [Ume81, Thm.2] to arbitrary dimension:

5.1. Lemma. Let $Y$ be a projective scheme of pure dimension $m \geq 2$ such that the dualising sheaf is trivial. Suppose that there exists an irreducible component $Y_{1} \subset Y$ having two points $\left\{p_{1}, p_{2}\right\}$ which are not contained in any other component of $Y$ such that $Y_{1}$ is normal near $\left\{p_{1}, p_{2}\right\}$, and that $\left\{p_{1}, p_{2}\right\}$ are isolated non-klt points. Let $\mu: Y^{\prime} \rightarrow Y$ be the birational morphism defined by the canonical modification of $Y_{1}$ in the points $p_{1}$ and $p_{2}$. If $Y$ is log-canonical in $p_{1}$ and $p_{2}$, then $h^{m-1}\left(Y^{\prime}, \mathcal{O}_{Y^{\prime}}\right) \geq 1$.

Proof. The image of the $\mu$-exceptional locus equals $\left\{p_{1}, p_{2}\right\}$, so the higher direct image sheaves $R^{j} \mu_{*} \mathcal{O}_{Y^{\prime}}$ have support in a finite set. Our goal is to prove that we have

$$
h^{0}\left(Y, R^{m-1} \mu_{*} \mathcal{O}_{Y^{\prime}}\right) \geq 2 .
$$

Assuming this for the time being, let us see how to conclude by a Leray spectral sequence computation: since the sheaves $R^{j} \mu_{*} \mathcal{O}_{Y^{\prime}}$ have no higher cohomology for $j>0$ we see that

$$
H^{0}\left(Y, R^{m-1} \mu_{*} \mathcal{O}_{Y^{\prime}}\right)=E_{2}^{0, m-1}=E_{m}^{0, m-1}
$$

and

$$
\mathbb{C} \cong H^{0}\left(Y, \omega_{Y}\right) \cong H^{m}\left(Y, \mathcal{O}_{Y}\right)=E_{2}^{m, 0}=E_{m}^{m, 0} .
$$

By (6) the first space has dimension at least two. Thus the kernel of the map

$$
d_{m}: E_{m}^{0, m-1} \rightarrow E_{m}^{m, 0}
$$

has dimension at least one, hence $\operatorname{dim} E_{m+1}^{0, m-1}=\operatorname{dim} E_{\infty}^{0, m-1}>0$. Since the map $H^{m-1}\left(Y^{\prime}, \mathcal{O}_{Y^{\prime}}\right) \rightarrow E_{\infty}^{0, m-1}$ is surjective the statement follows.

For the proof of (6) note first that the claim is local in a neighbourhood of the points $\left\{p_{1}, p_{2}\right\}$. Thus we can suppose without loss of generality that $Y$ is a normal variety with trivial canonical divisor such that $\operatorname{Nklt}(Y)=\left\{p_{1}, p_{2}\right\}$ and $\mu: Y^{\prime} \rightarrow Y$ is the canonical modification. Since $Y^{\prime}$ has canonical, hence rational singularities, we can replace it with a desingularisation, which for simplicity's sake we denote by the same letter. Since $K_{Y}$ is Cartier we can write

$$
K_{Y^{\prime}} \simeq \mu^{*} K_{Y}-E+F
$$

where $E$ is a reduced divisor mapping surjectively onto $\operatorname{Nklt}(Y)$ and $F$ is an effective divisor such that $E$ and $F$ have no common components. In particular $\omega_{E} \simeq \mathcal{O}_{E}(F)$ is effective. By Kovács' vanishing theorem [Kov11, Cor.6.6] we have $R^{j} \mu_{*} \mathcal{O}_{Y^{\prime}}(-E)=0$ for all $j>0$, hence

$$
R^{j} \mu_{*} \mathcal{O}_{Y^{\prime}} \simeq R^{j}\left(\left.\mu\right|_{E}\right)_{*} \mathcal{O}_{E}
$$

for all $j>0$. By duality (in the sense of [Har77, III, Sect.7, Defn.,p.241]) we have

$$
H^{m-1}\left(E, \mathcal{O}_{E}\right) \cong H^{0}\left(E, \omega_{E}\right) \text {. }
$$


We have seen that $\omega_{E}$ is effective. Since $E$ has at least two connected components the inequality (6) follows.

5.2. Definition. Let $X$ be a Fano variety of dimension $n$ and index $k$ with $l c$ singularities, and let $H$ be a Cartier divisor such that $-K_{X} \simeq k H$. We say that $X$ admits a ladder if there exist $k$ general divisors $D_{1}, \ldots, D_{k}$ in $|H|$ such that for all $i \in\{1, \ldots, k\}$ the intersection

$$
Z_{i}:=D_{1} \cap \ldots \cap D_{i}
$$

is a normal variety of dimension $n-k$ with lc singularities such that

$$
\operatorname{Nklt}\left(Z_{i}\right)=\operatorname{Nklt}(X) \cap D_{1} \cap \ldots \cap D_{i} .
$$

5.3. Proposition. Let $X$ be a Fano variety of dimension $n$ and index $k$ with $l c$ singularities, and let $H$ be a Cartier divisor such that $-K_{X} \simeq k H$. Suppose that $\left(\operatorname{Nklt}(X), \mathcal{O}_{\operatorname{Nklt}(X)}(H)\right)$ is a quadric of dimension $k$. If $k>1$ suppose also that $X$ admits a ladder. Then we have

$$
h^{n-k-1}\left(X^{\prime}, \mathcal{O}_{X^{\prime}}\right) \neq 0,
$$

where $X^{\prime} \rightarrow X$ is the canonical modification. Moreover the base of the MRCfibration $X^{\prime} \rightarrow Z$ has dimension $n-k-1>0$.

Proof. Note first that by the Nadel vanishing theorem the restriction map

$$
H^{0}\left(X, \mathcal{O}_{X}(H)\right) \rightarrow H^{0}\left(\operatorname{Nklt}(X), \mathcal{O}_{\text {Nklt }(X)}(H)\right)
$$

is surjective. Since $\mathcal{O}_{\operatorname{Nklt}(X)}(H)$ is globally generated, this implies that $|H|$ is globally generated near $\operatorname{Nklt}(X)$. In particular if $Y \in|H|$ is a general divisor, then we can apply the adjunction formula BS95. Lemma 1.7.6] to see that $\omega_{Y} \simeq$ $\mathcal{O}_{Y}((k-1) H)$. Moreover the intersection

$$
Y \cap \operatorname{Nklt}(X)
$$

is a quadric of dimension $k-1$ and $Y \cap \operatorname{Nklt}(X)$ is a connected component of the non-klt locus of $Y^{3}$. Let $\mu: X^{\prime} \rightarrow X$ be the canonical modification, then the strict transform $Y^{\prime}$ of $Y$ coincides with the total transform, hence we have $Y^{\prime} \simeq \mu^{*} H$. Moreover the birational map $\left.\mu\right|_{Y^{\prime}}: Y^{\prime} \rightarrow Y$ is the canonical modification of $Y$ along $Y \cap \operatorname{Nklt}(X)$.

Since $\mu^{*} H$ is nef and big, we have

$$
H^{j}\left(X^{\prime}, \mathcal{O}_{X^{\prime}}\left(-\mu^{*} H\right)\right)=0
$$

for all $j \leq n-1$ by [KM98, Thm.2.70]. Since $n-k-1 \leq n-2$ we obtain

$$
H^{n-k-1}\left(X^{\prime}, \mathcal{O}_{X^{\prime}}\right) \rightarrow H^{n-k-1}\left(Y^{\prime}, \mathcal{O}_{Y^{\prime}}\right) \text {. }
$$

We will now conclude by induction: if $k=1$, then $\omega_{Y}$ is trivial and $\left.\mu\right|_{Y^{\prime}}: Y^{\prime} \rightarrow Y$ satisfies the conditions of Lemma 5.1. Thus we have $H^{n-2}\left(Y^{\prime}, \mathcal{O}_{Y^{\prime}}\right) \neq 0$. If $k \geq 2$, then by hypothesis the Fano variety $X$ admits a ladder $D_{1}=Y, D_{2}, \ldots, D_{k}$, so $Y$ has lc singularities, $\operatorname{Nklt}(Y)$ is a quadric and the divisors

$$
D_{i} \cap Y \in|H|_{Y} \mid
$$

define a ladder on $Y$. Thus the induction hypothesis applies to $Y$.

\footnotetext{
${ }^{3}$ The divisor $Y$ may have several irreducible components, but by Bertini's theorem there exists a unique irreducible component that intersects $\operatorname{Nklt}(X)$. Note that this component is normal near $\operatorname{Nklt}(X)$ so it makes sense to speak of the non-klt locus.
} 
This also implies that the base of the MRC-fibration has dimension at least $n-k-1$. In order to see that equality holds let us first deal with the case $k=1$. As above let $Y \in|H|$ be a general divisor, and let $Y_{1} \subset Y$ be the unique irreducible component that meets $\operatorname{Nklt}(X)$. Let $Y_{1}^{\prime}\left(\right.$ resp. $\left.Y^{\prime}\right)$ be the strict transform under the canonical modification $\mu$. Since $\operatorname{Nklt}(X) \cap Y=\operatorname{Nklt}(X) \cap Y_{1}$ is not empty and $\omega_{Y} \simeq \mathcal{O}_{Y}$ we see that $\omega_{Y^{\prime}}$ is antieffective. Moreover we have a map $\omega_{Y_{1}^{\prime}} \rightarrow \omega_{Y^{\prime}} \otimes \mathcal{O}_{Y_{1}}$ that is an isomorphism in the generic point of $Y_{1}^{\prime}$, so $\omega_{Y_{1}^{\prime}}$ is antieffective. Thus we see that $Y_{1}^{\prime}$ is uniruled. Since $Y_{1}^{\prime}$ is general (note that by (7) we have $h^{0}\left(X, \mathcal{O}_{X}(H)\right) \geq 3$ ), it is not contracted by the MRC-fibration $X^{\prime} \rightarrow Z$. Since $Z$ is not uniruled GHS03, we obtain $\operatorname{dim} Z<\operatorname{dim} Y_{1}^{\prime}=n-1$.

If $k>1$ then by hypothesis we can consider the complete intersection $Z_{n-k}$ defined in Definition 5.2. Then $K_{Z_{n-k}}$ is trivial and by what precedes its strict transform $Z_{n-k}^{\prime} \subset X^{\prime}$ is uniruled. As in the case $k=1$ we obtain $\operatorname{dim} Z<\operatorname{dim} Z_{n-k}=$ $n-k$.

Theorem 1.6 is now an immediate consequence:

Proof of Theorem 1.6. The dimension of the base of the MRC-fibration is a birational invariant for varieties with canonical singularities [HM07, so by Proposition 5.3 we have $\operatorname{dim} Z=n-2$. Thus we can conclude with Proposition 4.2 .

For Fano varieties with high index we can verify the ladder condition in Proposition 5.3

5.4. Proposition. Let $X$ be a Fano variety of dimension $n$ and index $k \geq n-3$ with lc singularities, and let $H$ be a Cartier divisor such that $-K_{X} \simeq k H$. Suppose that $\operatorname{dim} \operatorname{Nklt}(X)=k$. Let $Y \in|H|$ be a general divisor. Then $Y$ is a normal variety with lc singularities such that

$$
\operatorname{Nklt}(Y)=\operatorname{Nklt}(X) \cap Y \text {. }
$$

Proof. By the Nadel vanishing theorem the restriction map

$$
H^{0}\left(X, \mathcal{O}_{X}(H)\right) \rightarrow H^{0}\left(\operatorname{Nklt}(X), \mathcal{O}_{\text {Nklt }(X)}(H)\right)
$$

is surjective. By Theorem 1.4 we know that $\mathcal{O}_{\mathrm{Nklt}(X)}(H)$ is globally generated, so we have

$$
\operatorname{Bs}|H| \cap \operatorname{Nklt}(X)=\emptyset .
$$

We claim that the pair $(X \backslash \operatorname{Nklt}(X), Y \backslash \operatorname{Nklt}(X))$ is plt. Assuming this for the time being, let us see how to conclude. Since the pair $(X \backslash \operatorname{Nklt}(X), Y \backslash \operatorname{Nklt}(X))$ is plt we know by inversion of adjunction Kol97, Thm.7.5.1] that $Y \backslash \operatorname{Nklt}(X)$ has canonical singularities. Since $\left.H\right|_{X \backslash \mathrm{Bs}|H|}$ is a free linear system, a general $Y \in|H|$ is normal by [BS95, Thm.1.7.1] and $(X \backslash \mathrm{Bs}|H|, Y \backslash \mathrm{Bs}|H|)$ is an lc pair. Using the adjunction formula [BS95, Lemma 1.7.6] we see that for a general $Y \in|H|$ we have $-\left.K_{Y} \simeq(k-1) H\right|_{Y}$. By inversion of adjunction $Y$ has lc singularities Kaw07, Thm. p.130] and

$$
\operatorname{Nklt}(Y)=\operatorname{Nklt}(X) \cap Y \text {. }
$$

Proof of the claim. We will deal with the case $k=n-3$, the other cases being simpler. We follow the argument of Flo13, Thm.1.1]: note that $X \backslash \operatorname{Nklt}(X)$ has canonical singularities. We argue by contradiction and suppose that the pair 
$(X \backslash \operatorname{Nklt}(X), Y \backslash \operatorname{Nklt}(X))$ is not plt. Then there exists a $0<c \leq 1$ and an irreducible variety $W \subset \mathrm{Bs}|H|$ such that the pair $(X \backslash \operatorname{Nklt}(X), c(Y \backslash \operatorname{Nklt}(X)))$ is properly lc and $W$ a minimal lc centre. By [Fuj11, Thm.2.2] the restriction map

$$
H^{0}\left(X, \mathcal{O}_{X}(H)\right) \rightarrow H^{0}\left(W, \mathcal{O}_{W}(H)\right)
$$

is surjective. Since $W$ is contained in the base locus this implies that $H^{0}\left(W, \mathcal{O}_{W}(H)\right)=0$. By Kawamata subadjunction (2) there exists an effective divisor $\Delta_{W}$ such that $\left(W, \Delta_{W}\right)$ is klt and

$$
\left.\left(K_{W}+\Delta_{W}\right) \sim_{\mathbb{Q}}\left(K_{X}+c Y\right)\right|_{W} \sim_{\mathbb{Q}}-\left.(n-3-c) H\right|_{W} .
$$

If $\operatorname{dim} W \leq 2$ we apply [Kaw00, Prop.4.1] to see that $h^{0}\left(W, \mathcal{O}_{W}(H)\right) \neq 0$, a contradiction. If $\operatorname{dim} W \geq 3$ and $n-3-c>\operatorname{dim} W-3$, then $\left(W, B_{W}\right)$ is $\log$ Fano of index at least $n-3-c$. By Kaw00, Theorem 5.1] this implies $h^{0}\left(W, \mathcal{O}_{W}(H)\right) \neq 0$, a contradiction. Thus we are left with the case when $\operatorname{dim} W \geq 3$ and $\operatorname{dim} W \geq n-c$. Since $c \leq 1$ this implies $\operatorname{dim} W=n-1$. Since the centre $W$ is minimal we know by [Flo13, Lemma 2.7] that $c<1 / 2$. Thus we have $\operatorname{dim} W \geq n-1 / 2$, a contradiction.

\section{EXAMPLES}

In [BS87, §2] the first-named author and Sommese observed that for a Fano threefold of index one with $\operatorname{dim} \operatorname{Nklt}(X)=1$, the non-klt locus consists of rational curves. They also ask how far $X$ can deviate from being a generalised cone with $\operatorname{Nklt}(X)$ as its vertex. In this section we answer this question: all the classification results of Section 4 are effective, so in many cases $X$ is far from being a generalised cone.

6.1. Example. Let $Y$ be a projective manifold with trivial canonical divisor, and let $L$ be a very ample line bundle on $Y$. Set

$$
X^{\prime}:=\mathbb{P}\left(\mathcal{O}_{Y}^{\oplus k} \oplus L\right),
$$

then we have

$$
K_{X^{\prime}}=-k \zeta-\left(\zeta-\varphi^{*} L\right),
$$

where $\zeta$ is the tautological divisor on $X^{\prime}$. We have $\zeta-\varphi^{*} L=E$ where $E \cong Y \times \mathbb{P}^{k-1}$ is the divisor defined by the quotient

$$
\mathcal{O}_{Y}^{\oplus k} \oplus L \rightarrow \mathcal{O}_{Y}^{\oplus k}
$$

The divisor $\zeta$ is globally generated and defines a birational morphism $\mu: X^{\prime} \rightarrow X$ that contracts $E$ onto a $\mathbb{P}^{k-1}$. Using the canonical bundle formula above we see that $X$ is a Fano variety of index $k$ with lc singularities, moreover the non-klt locus is a linear $\mathbb{P}^{k-1}$.

6.2. Example. Let $Y$ be a projective manifold admitting a fibration $\psi: Y \rightarrow \mathbb{P}^{1}$ such that $\mathcal{O}_{Y}\left(-K_{Y}\right) \simeq \psi^{*} \mathcal{O}_{\mathbb{P}^{1}}(1)$. Then set $X^{\prime}:=\mathbb{P}\left(A \oplus \psi^{*} \mathcal{O}_{\mathbb{P}^{1}}(1) \oplus \mathcal{O}_{Y}^{\oplus k-1}\right)$ where $A$ is a very ample line bundle on $Y$. Let $\zeta$ be the tautological divisor on $X^{\prime}$, then $\zeta$ is globally generated and defines a birational morphism $\mu: X^{\prime} \rightarrow X$ contracting the divisor $E$ corresponding to the quotient

$$
A \oplus \psi^{*} \mathcal{O}_{\mathbb{P}^{1}}(1) \oplus \mathcal{O}_{Y}^{\oplus k-1} \rightarrow \psi^{*} \mathcal{O}_{\mathbb{P}^{1}}(1) \oplus \mathcal{O}_{Y}^{\oplus k-1} \simeq \psi^{*}\left(\mathcal{O}_{\mathbb{P}^{1}}(1) \oplus \mathcal{O}_{\mathbb{P}^{1}}^{\oplus k-1}\right)
$$

onto a $\mathbb{P}^{k}$. Using the canonical bundle formula one easily sees that $X$ is Fano of index $k$ with lc singularities and $\left(\operatorname{Nklt}(X), \mathcal{O}_{\text {Nklt }(X)}(H)\right) \cong\left(\mathbb{P}^{k}, \mathcal{O}_{\mathbb{P}^{k}}(1)\right)$, where $-K_{X} \simeq k H$. 
6.3. Example. Let $X$ be a Fano variety of index $m$ constructed as in Example 6.1. and let $-K_{X} \simeq m H$ with $H$ a Cartier divisor on $X$. Set $k=m-1$. By the Nadel vanishing theorem the restriction map

$$
H^{0}\left(X, \mathcal{O}_{X}(2 H)\right) \rightarrow H^{0}\left(\operatorname{Nklt}(X), \mathcal{O}_{\text {Nklt }(X)}(2 H)\right)
$$

is surjective. Fix now a quadric $Q \subset \mathbb{P}^{k} \cong \operatorname{Nklt}(X)$ and fix a general divisor $B$ in the linear system $|2 H|$ such that $B \cap \operatorname{Nklt}(X)=Q$. Denote by

$$
\pi: \tilde{X} \rightarrow X
$$

the cyclic covering of degree two branched along $B$. By the ramification formula we see that $-K_{\widetilde{X}}=\pi^{*} k H$, so $\widetilde{X}$ is a Fano variety of index $k$. Using again the ramification formula we know by [KM98, Prop.5.20] that $\widetilde{X}$ has lc singularities if and only if the pair $\left(X, \frac{1}{2} B\right)$ is lc. Since $B$ is general this is clear in the complement of $\operatorname{Nklt}(X)$, moreover by inversion of adjunction [Hac12, Thm.0.1] the pair $\left(X, \frac{1}{2} B\right)$ is lc near $\operatorname{Nklt}(X)$ if and only if $\left(\mathbb{P}^{k}, \frac{1}{2} Q\right)$ is lc. Since $Q$ is a quadric this is easily checked. The restriction of $\pi$ to $\operatorname{Nklt}(\widetilde{X}) \cong \mathbb{P}^{k}$ induces a two-to-one cover

$$
\operatorname{Nklt}(\tilde{X}) \rightarrow \operatorname{Nklt}(X)
$$

that ramifies exactly along $Q=B \cap \operatorname{Nklt}(X)$. Since $Q$ is a quadric in $\mathbb{P}^{k}$, we see that $\operatorname{Nklt}(\widetilde{X})$ is a quadric, the singularities depending on the singularities of $Q$. Let $X^{\prime} \rightarrow Y$ be the projective bundle dominating $X$ (cf. Example 6.1). Then $X^{\prime} \times_{X} \widetilde{X} \rightarrow Y$ is a quadric bundle dominating $\widetilde{X}$.

6.4. Example. Let $Y$ be a projective manifold with trivial canonical divisor. Let $L$ be a very ample line bundle on $Y$ and set

$$
B^{\prime}:=\mathbb{P}\left(L \oplus \mathcal{O}_{Y}^{\oplus 2}\right) .
$$

Denote by $\eta$ the tautological divisor on $B^{\prime}$, then $2 \eta$ is globally generated and defines a birational morphism $\nu: B^{\prime} \rightarrow B$ onto a normal projective variety contracting the divisor $D$ corresponding to the quotient

$$
L \oplus \mathcal{O}_{Y}^{\oplus 2} \rightarrow \mathcal{O}_{Y}^{\oplus 2}
$$

onto a $\mathbb{P}^{1}$. Using the canonical bundle formula for $B^{\prime}$ we see that

$$
K_{B^{\prime}} \simeq-2 \eta-D \simeq \nu^{*} K_{B}-D,
$$

so $B$ has lc singularities and $\left(\operatorname{Nklt}(B), \mathcal{O}_{\text {Nklt }(B)}\left(-K_{B}\right)\right) \cong\left(\mathbb{P}^{1}, \mathcal{O}_{\mathbb{P}^{1}}(2)\right)$. For some integer $k \geq 2$ we set

$$
X^{\prime}:=\mathbb{P}\left(\mathcal{O}_{B^{\prime}}(2 \eta) \oplus \mathcal{O}_{B^{\prime}}^{\oplus k-1}\right),
$$

and denote by $\zeta$ the tautological divisor on $\varphi: X^{\prime} \rightarrow B^{\prime}$. The divisor $\zeta$ is globally generated and defines a birational morphism $\mu: X^{\prime} \rightarrow X$. If we restrict $\mu$ to $\varphi^{-1}\left(\mathbb{P}^{2}\right)$ where $\mathbb{P}^{2}$ is a fibre of $B^{\prime} \rightarrow Y$, the image is a generalised cone of dimension $k+1$ over the Veronese surface $\left(\mathbb{P}^{2}, \mathcal{O}_{\mathbb{P}^{2}}(2)\right)$. If we restrict $\mu$ furthermore to $\varphi^{-1}\left(\mathbb{P}^{2} \cap D\right)$, the image is the generalised cone of dimension $k$ over the line $\mathbb{P}^{2} \cap D$, but polarised by $\mathcal{O}_{\mathbb{P}^{2}}(2)$, so we get a quadric $Q$ of dimension $k$ such that the singular locus has dimension $k-2$. Since $D \simeq \mathbb{P}\left(\mathcal{O}_{Y}^{\oplus 2}\right)$, a straightforward computation shows that

$$
\zeta^{k+1} \cdot \varphi^{*} D=0 \text { in } H^{2 k+4}\left(X^{\prime}, \mathbb{R}\right),
$$

\footnotetext{
${ }^{4}$ Note that by the generality assumption the divisor $B$ is reduced even if $Q$ is not reduced. In particular $\widetilde{X}$ is irreducible.
} 
so the divisor $E:=\varphi^{*} D$ is contracted by $\mu$ onto the $k$-dimensional quadric $Q$. Using the canonical bundle formula we see that

$$
K_{X^{\prime}}=-k \zeta-E=\mu^{*} K_{X}-E,
$$

so $X$ is a Fano variety of index $k$ having lc singularities and $\operatorname{Nklt}(X) \cong Q$. Note also that $\mu$ factors through a birational morphism $p: \Gamma \rightarrow X$ where $\Gamma$ is a normal projective variety admitting a locally trivial fibration $q: \Gamma \rightarrow Z$ such that the polarised fibre is the generalised cone of dimension $k+1$ over the Veronese surface $\left(\mathbb{P}^{2}, \mathcal{O}_{\mathbb{P}^{2}}(2)\right)$.

6.5. Example. For some $k \geq 3$, let $Y \subset \mathbb{P}^{k-1}$ be a smooth hypersurface of degree $k$, so $Y$ is a Calabi-Yau manifold. We set

$$
B^{\prime}:=\mathbb{P}\left(\mathcal{O}_{Y}(1) \oplus \mathcal{O}_{Y}\right),
$$

and denote by $\eta$ the tautological divisor on $B^{\prime}$. Then $\eta$ is globally generated and defines a birational morphism $\nu: B^{\prime} \rightarrow B$ where $B$ is the cone over $Y$. We have $K_{B^{\prime}} \simeq \nu^{*} K_{B}-D$, where $D$ is the divisor corresponding to the quotient

$$
\mathcal{O}_{Y}(1) \oplus \mathcal{O}_{Y} \rightarrow \mathcal{O}_{Y}
$$

Note that $B$ is a hypersurface of degree $k$ in $\mathbb{P}^{k}$, so $B$ is Cohen-Macaulay and has exactly one non-klt point, the vertex of the cone. The anticanonical sheaf $\mathcal{O}_{B}\left(-K_{B}\right) \simeq \mathcal{O}_{B}(1)$ is globally generated and $h^{0}\left(B, \mathcal{O}_{B}\left(-K_{B}\right)\right)=k+1$. We define $W^{*}$ to be the vector bundle of rank $k$ that is the kernel of the evaluation map $\mathcal{O}_{B}^{\oplus k+1} \rightarrow \mathcal{O}_{B}\left(-K_{B}\right)$. Dualizing we obtain an exact sequence

$$
0 \rightarrow \mathcal{O}_{B}\left(K_{B}\right) \rightarrow \mathcal{O}_{B}^{\oplus k+1} \rightarrow W \rightarrow 0,
$$

so $W$ is globally generated. Moreover by a singular version of Kodaira vanishing [KSS10, Cor.6.6] we have $H^{1}\left(B, \mathcal{O}_{B}\left(K_{B}\right)\right)=0$, so we get $h^{0}(B, W)=k+1$.

Set now $W^{\prime}:=\nu^{*} W$ and let $A$ be the pull-back of a very ample Cartier divisor on $B$. We set

$$
X^{\prime}:=\mathbb{P}\left(\mathcal{O}_{B^{\prime}}(A) \oplus W^{\prime}\right),
$$

and denote by $\zeta$ the tautological divisor on $\varphi: X^{\prime} \rightarrow B^{\prime}$. The divisor $\zeta$ is globally generated and defines a birational morphism $\mu: X^{\prime} \rightarrow X$. Denote by $E \subset X^{\prime}$ the divisor defined by the quotient

$$
\mathcal{O}_{B^{\prime}}(A) \oplus W^{\prime} \rightarrow W^{\prime}
$$

Then by the canonical bundle formula

$K_{X^{\prime}} \simeq \varphi^{*}\left(K_{B^{\prime}}+A+\operatorname{det} W^{\prime}\right)-(k+1) \zeta=-k \zeta-\left(\zeta-\varphi^{*} A\right)+\varphi^{*}\left(K_{B^{\prime}}+\operatorname{det} W^{\prime}\right)$.

By construction we have $\zeta-\varphi^{*} A \simeq E$ and $K_{B^{\prime}}+\operatorname{det} W^{\prime} \simeq-D$, so we get

$$
K_{X^{\prime}} \simeq-k \zeta-E-\varphi^{*} D \text {. }
$$

Since $D$ is contracted by $\nu$, the restriction of $\mathcal{O}_{B^{\prime}}(A) \oplus W^{\prime}$ to $D$ is isomorphic to $\mathcal{O}_{D}^{\oplus k+1}$, so $\varphi^{*} D$ is contracted by $\mu$ onto a $\mathbb{P}^{k}$. The divisor $E$ is isomorphic to $\mathbb{P}\left(W^{\prime}\right)$ and by construction $h^{0}\left(B^{\prime}, W^{\prime}\right)=k+1$, so $E$ is also contracted onto a $\mathbb{P}^{k}$. Thus $X$ is a Fano variety of index $k$ with lc singularities such that $\operatorname{Nklt}(X)$ is a reducible quadric. 
6.6. Example. Let $Y$ be a projective manifold with trivial canonical divisor and set $B:=Y \times \mathbb{P}^{1}$. Let $A$ be a very ample Cartier divisor on $B$ and set

$$
X^{\prime}:=\mathbb{P}\left(\mathcal{O}_{B}(A) \oplus \mathcal{O}_{B}\left(-K_{B}\right) \oplus \mathcal{O}_{B}^{\oplus k-1}\right) .
$$

Denote by $\zeta$ the tautological divisor on $\varphi: X^{\prime} \rightarrow B$. The divisor $\zeta$ is globally generated and defines a birational morphism $\mu: X^{\prime} \rightarrow X$. Moreover if $E \subset X^{\prime}$ is the divisor defined by the quotient

$$
\mathcal{O}_{B}(A) \oplus \mathcal{O}_{B}\left(-K_{B}\right) \oplus \mathcal{O}_{B}^{\oplus k-1} \rightarrow \mathcal{O}_{B}\left(-K_{B}\right) \oplus \mathcal{O}_{B}^{\oplus k-1},
$$

then $E$ is contracted by $\mu$ onto a quadric $Q$ of dimension $k$ that is singular along a subvariety of dimension $k-2$. By the canonical bundle formula we see that

$$
K_{X^{\prime}}=-k \zeta-E=\mu^{*} K_{X}-E,
$$

so $X$ is a Fano variety of index $k$ having lc singularities and $\operatorname{Nklt}(X) \cong Q$.

Analogously, if we set

$$
X^{\prime}:=\mathbb{P}\left(\mathcal{O}_{B}(A) \oplus \mathcal{O}_{B}\left(-\frac{1}{2} K_{B}\right)^{\oplus 2} \oplus \mathcal{O}_{B}^{\oplus k-2}\right),
$$

the same properties hold; in this case the quadric is singular along a subvariety of dimension $k-3$.

\section{REFERENCES}

[AD12] Carolina Araujo and Stéphane Druel. On codimension 1 del Pezzo foliations on varieties with mild singularities. arXiv preprint, 1210.4013, 2012.

[Amb04] Florin Ambro. Shokurov's boundary property. J. Differential Geom., 67(2):229-255, 2004.

[Amb05] Florin Ambro. The moduli b-divisor of an lc-trivial fibration. Compos. Math., 141(2):385-403, 2005.

[Amb11] Florin Ambro. Basic properties of log canonical centers. In Classification of algebraic varieties, EMS Ser. Congr. Rep., pages 39-48. Eur. Math. Soc., Zürich, 2011.

[And95] Marco Andreatta. Some remarks on the study of good contractions. Manuscripta Math., 87(3):359-367, 1995.

[AW93] Marco Andreatta and Jarosław A. Wiśniewski. A note on nonvanishing and applications. Duke Math. J., 72(3):739-755, 1993.

[BCHM10] Caucher Birkar, Paolo Cascini, Christopher D. Hacon, and James McKernan. Existence of minimal models for varieties of log general type. J. Amer. Math. Soc., 23(2):405-468, 2010.

[BHPVdV04] Wolf P. Barth, Klaus Hulek, Chris A. M. Peters, and Antonius Van de Ven. Compact complex surfaces, volume 4 of Ergebnisse der Mathematik und ihrer Grenzgebiete. 3. Folge. Springer-Verlag, Berlin, second edition, 2004.

[BP11] Amaël Broustet and Gianluca Pacienza. Rational connectedness modulo the non-nef locus. Comment. Math. Helv., 86(3):593-607, 2011.

[BS87] Mauro C. Beltrametti and Andrew J. Sommese. A criterion for a variety to be a cone. Comment. Math. Helv., 62(3):417-422, 1987.

[BS95] Mauro C. Beltrametti and Andrew J. Sommese. The adjunction theory of complex projective varieties, volume 16 of de Gruyter Expositions in Mathematics. Walter de Gruyter \& Co., Berlin, 1995.

[Deb01] Olivier Debarre. Higher-dimensional algebraic geometry. Universitext. SpringerVerlag, New York, 2001.

[FG12] Osamu Fujino and Yoshinori Gongyo. On canonical bundle formulas and subadjunctions. Michigan Math. J., 61(2):255-264, 2012.

[Flo13] Enrica Floris. Fundamental divisors on Fano varieties of index $n-3$. Geom. Dedicata, 162:1-7, 2013. 
[Fuj90] Takao Fujita. Classification theories of polarized varieties, volume 155 of London Mathematical Society Lecture Note Series. Cambridge University Press, Cambridge, 1990.

[Fuj11] Osamu Fujino. Fundamental theorems for the log minimal model program. Publ. Res. Inst. Math. Sci., 47(3):727-789, 2011.

[GHS03] Tom Graber, Joe Harris, and Jason Starr. Families of rationally connected varieties. J. Amer. Math. Soc., 16(1):57-67 (electronic), 2003.

[Hac12] Christopher Hacon. On log canonical inversion of adjunction. arXiv preprint, 1202.0491, 2012.

[Har77] Robin Hartshorne. Algebraic geometry. Springer-Verlag, New York, 1977. Graduate Texts in Mathematics, No. 52

[HM07] Christopher D. Hacon and James Mckernan. On Shokurov's rational connectedness conjecture. Duke Math. J., 138(1):119-136, 2007.

[HN13] Andreas Höring and Carla Novelli. Mori contractions of maximal length. Publ. Res. Inst. Math. Sci., 49(1):215-228, 2013.

[Hör13] Andreas Höring. Twisted cotangent sheaves and a Kobayashi-Ochiai theorem for foliations. arXiv preprint, 1307.3449, 2013.

[Ish91] Shihoko Ishii. Quasi-Gorenstein Fano 3-folds with isolated nonrational loci. Compositio Math., 77(3):335-341, 1991.

[Ish94] Shihoko Ishii. On Fano 3-folds with non-rational singularities and two-dimensional base. Abh. Math. Sem. Univ. Hamburg, 64:249-277, 1994.

[Kaw97] Yujiro Kawamata. On Fujita's freeness conjecture for 3-folds and 4-folds. Math. Ann., 308(3):491-505, 1997.

[Kaw98] Yujiro Kawamata. Subadjunction of log canonical divisors. II. Amer. J. Math., 120(5):893-899, 1998.

[Kaw00] Yujiro Kawamata. On effective non-vanishing and base-point-freeness. Asian J. Math., 4(1):173-181, 2000. Kodaira's issue.

[Kaw07] Masayuki Kawakita. Inversion of adjunction on $\log$ canonicity. Invent. Math., 167(1):129-133, 2007.

[KK10] János Kollár and Sándor J. Kovács. Log canonical singularities are Du Bois. J. Amer. Math. Soc., 23(3):791-813, 2010.

[KM98] János Kollár and Shigefumi Mori. Birational geometry of algebraic varieties, volume 134 of Cambridge Tracts in Mathematics. Cambridge University Press, Cambridge, 1998. With the collaboration of C. H. Clemens and A. Corti.

[Kol97] János Kollár. Singularities of pairs. In Algebraic geometry-Santa Cruz 1995, volume 62 of Proc. Sympos. Pure Math., pages 221-287. Amer. Math. Soc., Providence, RI, 1997.

[Kol11] János Kollár. Sources of log-canonical centers. arXiv, 1107.2863, 2011.

[Kol13] János Kollár. Singularities of the minimal model program, volume 200 of Cambridge Tracts in Mathematics. Cambridge University Press, Cambridge, 2013. With a collaboration of Sándor Kovács.

[Kov11] Sándor J. Kovács. Du Bois pairs and vanishing theorems. Kyoto J. Math., 51(1):4769, 2011.

[KSS10] Sándor J. Kovács, Karl Schwede, and Karen E. Smith. The canonical sheaf of Du Bois singularities. Adv. Math., 224(4):1618-1640, 2010.

[Kwc92] János Kollár (with 14 coauthors). Flips and abundance for algebraic threefolds. Société Mathématique de France, Paris, 1992. Papers from the Second Summer Seminar on Algebraic Geometry held at the University of Utah, Salt Lake City, Utah, August 1991, Astérisque No. 211 (1992).

[Ume81] Yumiko Umezu. On normal projective surfaces with trivial dualizing sheaf. Tokyo J. Math., 4(2):343-354, 1981.

Mauro C. Beltrametti, Dipartimento di Matematica, Università degli Studi di Genova, Via Dodecaneso 35, I-16146 Genova, Italy

E-mail address: beltrame@dima.unige.it 
Andreas Höring, Laboratoire de Mathématiques J.A. Dieudonné, UMR 7351 CNRS, Université de Nice Sophia-Antipolis, 06108 Nice Cedex 02, France

E-mail address: hoering@unice.fr

Carla Novelli, Dipartimento di Matematica, Università degli Studi di Padova, via TrieSte 63, I-35121 Padova, Italy

E-mail address: novelli@math.unipd.it 